\title{
Spinal needle force monitoring during lumbar puncture using fiber Bragg grating force device
}

\author{
Shikha Ambastha \\ Sharath Umesh \\ Sundaresh Dabir \\ Sundarrajan Asokan
}




\title{
Spinal needle force monitoring during lumbar puncture using fiber Bragg grating force device
}

\author{
Shikha Ambastha, ${ }^{a}$ Sharath Umesh, ${ }^{a}$ Sundaresh Dabir, ${ }^{b}$ and Sundarrajan Asokan ${ }^{a, *}$ \\ andian Institute of Science, Department of Instrumentation and Applied Physics, Bangalore 560012, India \\ bri Sathya Sai Institute of Higher Medical Sciences, Department of Orthopaedics, Bangalore 560066, India
}

\begin{abstract}
A technique for real-time dynamic monitoring of force experienced by a spinal needle during lumbar puncture using a fiber Bragg grating (FBG) sensor is presented. The proposed FBG force device (FBGFD) evaluates the compressive force on the spinal needle during lumbar puncture, particularly avoiding the bending effect on the needle. The working principle of the FBGFD is based on transduction of force experienced by the spinal needle into strain variations monitored by the FBG sensor. FBGFD facilitates external mounting of a spinal needle for its smooth insertion during lumbar puncture without any intervention. The developed FBGFD assists study and analysis of the force required for the spinal needle to penetrate various tissue layers from skin to the epidural space; this force is indicative of the varied resistance offered by different tissue layers for the spinal needle traversal. Calibration of FBGFD is performed on a micro-universal testing machine for 0 to $20 \mathrm{~N}$ range with an obtained resolution of $0.021 \mathrm{~N}$. The experimental trials using spinal needles mounted on FBGFD are carried out on a human cadaver specimen with punctures made in the lumbar region from different directions. Distinct forces are recorded when the needle encounters skin, muscle tissue, and a bone in its traversing path. Real-time spinal needle force monitoring using FBGFD may reduce potentially serious complications during the lumbar puncture, such as overpuncturing of tissue regions, by impeding the spinal needle insertion at epidural space. $\odot$ 2016 Society of Photo-Optical Instrumentation Engineers (SPIE) [DOI: 10.1117/1.JBO.21.11.117002]
\end{abstract}

Keywords: spinal needle; force monitoring; lumbar puncture; fiber Bragg grating force device.

Paper 160532R received Aug. 3, 2016; accepted for publication Oct. 4, 2016; published online Nov. 7, 2016.

\section{Introduction}

Clinical practices mostly involve needle insertion procedures that require puncturing of skin tissues. A skilled medical practitioner penetrates the needle through different tissue layers in order to reach to the target location for treatment, optimizing the traverse path of the needle by virtue of his own sensory feel of the resistance force. Tissue properties for different individuals vary in terms of thickness, stiffness, and so on. In procedures such as biopsy and epidural anesthesia, needle positioning accuracy is highly essential. Study of biomedical interaction of a surgical needle for invasive diagnosis through tissue layers is an essential aspect for surgical applications. Critical cases of medical errors, such as accidental puncturing of vital organs during surgeries, committed by trainee surgeons and staff, need to be avoided. These can be prevented with a better understanding of needle interaction with tissue assisted by essential surgical skills training with advanced technical tools. ${ }^{1}$ Also, it is imperative to know the real-time positioning of the spinal needle during the lumbar puncture (LP) procedure which is utilized for several diagnostic and therapeutic medical practices involving collection of cerebrospinal fluid $(\mathrm{CSF})^{2}$ or to reduce increased intracranial pressure. The most common issue persisting in this methodology is post-dural-puncture headache. ${ }^{3}$

Earlier studies carried out on force feedback monitoring of needle insertion ${ }^{4-7}$ mainly focus on applications involving robotic surgery or minimally invasive surgeries using haptic feedback mechanism. ${ }^{8-15}$ Magill et al. demonstrated a mechanical

*Address all correspondence to: Sundarrajan Asokan, E-mail: sasokan@iap. iisc.ernet.in actuator-based technique that simulates a haptic experience similar to penetrating tissue with a needle using brushless motors supported force feedback system. ${ }^{16}$ A direct methodology of needle insertion force measurement has been illustrated for gelatine analogs, a bevel-tip needle mounted on a digital force gauge that provides the needle insertion forces used to model the consistency of the tissues in the lumbar region of the back. ${ }^{9}$ Another prototype for haptic force feedback monitoring on a human mannequin torso using a hardware- and software-based haptic device and simulation methodology was demonstrated by Gorman et al. ${ }^{2}$ A few other epidural hardware- and softwarebased simulators for force feedback monitoring are also reported. ${ }^{3,17-20}$ These techniques employ bulky sensors and complex system application for force estimation on spinal needle insertion. However, the above-mentioned methodologies do not provide a practical hand-held solution for force monitoring during spinal needle insertion.

Recently, FBG sensors are greatly in use for applications in the biomedical field due to their numerous advantages such as small size, chemical inertness, high sensitivity, ultrafast response, electrically passive operation, insensitivity to radio frequency, and immunity to electromagnetic interference. ${ }^{21-24}$ In their basic form, FBG sensors are sensitive to strain and temperature; with employment of various transduction techniques they can be used to sense a variety of parameters such as pressure, vibration, force, and flow. ${ }^{25-27}$

The present study describes a methodology of spinal needle force monitoring system during LP using fiber Bragg grating force device (FBGFD). The developed FBGFD transduces

$1083-3668 / 2016 / \$ 25.00$ @ 2016 SPIE 
the spinal needle interaction forces with tissue regions into strain variations that are acquired by the FBG sensor. A major advantage of using an FBG sensor in FBGFD is its compact dimension that fits in perfectly making it a rugged and portable device. The basic idea behind the design of FBGFD is to dynamically monitor the force experienced by a spinal needle during LP by which the region of penetration of the spinal needle may be evaluated. The prominent difficulty faced in force monitoring on the spinal needle is its bending effect. The major advantage of the developed FBGFD is its ability to measure the force experienced on the spinal needle by the elimination of bending effect.

\section{Materials and Methods}

\subsection{Fiber Bragg Grating Sensor}

An FBG sensor consists of a periodic modulation of the refractive index of the core of a single-mode photosensitive optical fiber along its axis. When a broadband light is launched into an optical fiber inscribed with FBG, one particular wavelength that satisfies the Bragg condition, given by Eq. (1), is reflected back and the remaining wavelengths are transmitted through the optical fiber. ${ }^{28}$ The reflected Bragg wavelength from the FBG is given as

$\lambda_{\mathrm{B}}=2 n_{\mathrm{eff}} \Lambda$.

Here, the Bragg resonance wavelength $\lambda_{\mathrm{B}}$ is the free space center wavelength of the input light that will be backreflected from the Bragg grating. $n_{\text {eff }}$ is the effective refractive index of the fiber and $\Lambda$ is the grating periodicity.

In the present work, phase mask technique is ${ }^{29-31}$ used for photoinscription of gratings ${ }^{32,33}$ by exposing the core of a photosensitive, germano-silicate fiber through an interference pattern created using $\mathrm{KrF}$ excimer UV laser of wavelength $248 \mathrm{~nm}$ (BraggStar industrial, TUI laser). FBG sensors with a gauge length of $3 \mathrm{~mm}$ are fabricated. The strain effect on an FBG sensor is expressed as

$\Delta \lambda_{\mathrm{B}}=\lambda_{\mathrm{B}}\left\{1-\frac{n_{\mathrm{eff}}^{2}}{2}\left[P_{12}-\nu\left(P_{11}-P_{12}\right)\right]\right\} \varepsilon$,

where $P_{11}$ and $P_{12}$ are components of the strain-optic tensor, $\nu$ is the Poisson's ratio, and $\varepsilon$ is the axial strain change. ${ }^{34}$ The strain sensitivity of an FBG inscribed in a germania-doped silica fiber is $\sim 1.2 \mathrm{pm} / \mu \varepsilon$. $^{35}$

As the temperature variations may also cause a shift in the Bragg wavelength, it is imperative to compensate for temperature variations (if any) while using the FBG sensor for strain measurements. In this work, experiments carried out are within a short duration of time and in controlled environmental conditions where temperature change was negligibly small thus the temperature effect on the FBG sensor is ignored.

\subsection{Design and Fabrication of Fiber Bragg Grating Force Device}

The FBGFD comprises two cylindrical segments made of stainless steel (top and bottom) of diameter $10 \mathrm{~mm}$ and length $15 \mathrm{~mm}$ each, joined together by means of a sensing bar of length $40 \mathrm{~mm}$ and diameter $0.9 \mathrm{~mm}$. The FBG sensor is positioned on the surface of the sensing bar such that the sensor grating length of $3 \mathrm{~mm}$ lies at the center along the length of the sensing bar. The FBG sensor is glued using industrial adhesive (Loctite 420) and allowed to cure for $30 \mathrm{~min}$. This assembly constitutes to form the sensing component of FBGFD as shown in Fig. 1(a). An opening on the top cylindrical segment is provided with threads in order to attach the spinal needle fixture. A spinal needle fixture with a head part of diameter $10 \mathrm{~mm}$ and tail part of diameter $4 \mathrm{~mm}$ is designed for holding the spinal needle. The spinal needle fixture facilitates a groove that is made to house the spinal needle with a depth of holding of $8 \mathrm{~mm}$ as shown in Fig. 1(b). The tail end of the spinal needle fixture can be screwed to the sensing component by the means of threads provided on it.

The assembly of the sensing component is housed inside a 70-mm-long hollow casing of inner diameter $10 \mathrm{~mm}$ and outer diameter $12 \mathrm{~mm}$. The inner diameter of the hollow tube is maintained such that the sensing component is housed perfectly inside it. This is done in order to restrict any lateral movement of the sensing component allowing it to move only axially. The length of the hollow tube is maintained so as to match the full longitudinal dimension of the sensing component. Both ends of the tube are closed using threaded caps provided with openings for the spinal needle fixture to attach on one side and for routing the optical fiber connecting FBG sensor on the other side. Thus, this mechanism ensures only 1-deg of freedom for the movement of the sensing component, i.e., only axial movement inside the hollow tube.

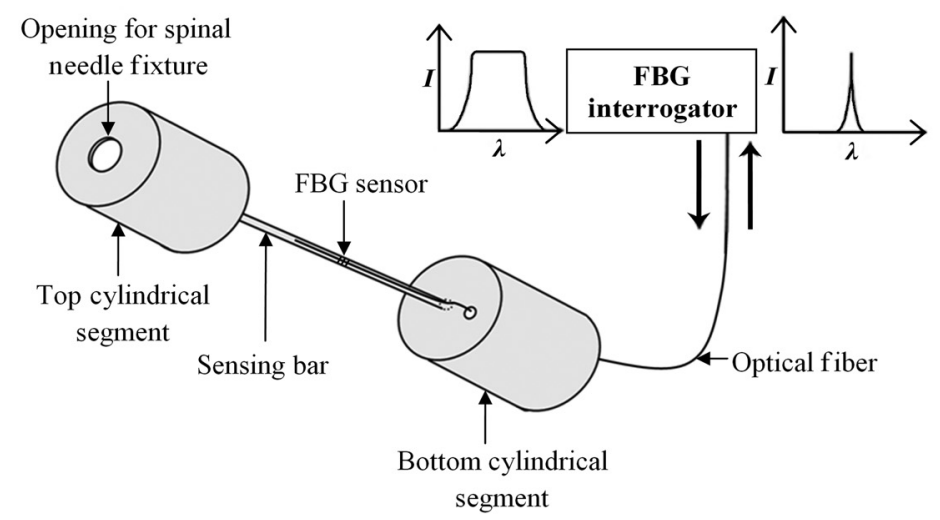

(a)

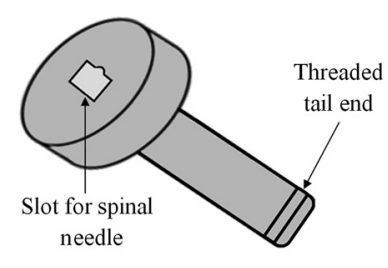

(b)

Fig. 1 Schematic of (a) FBGFD sensing bar with bonded FBG sensor and (b) spinal needle fixture. 


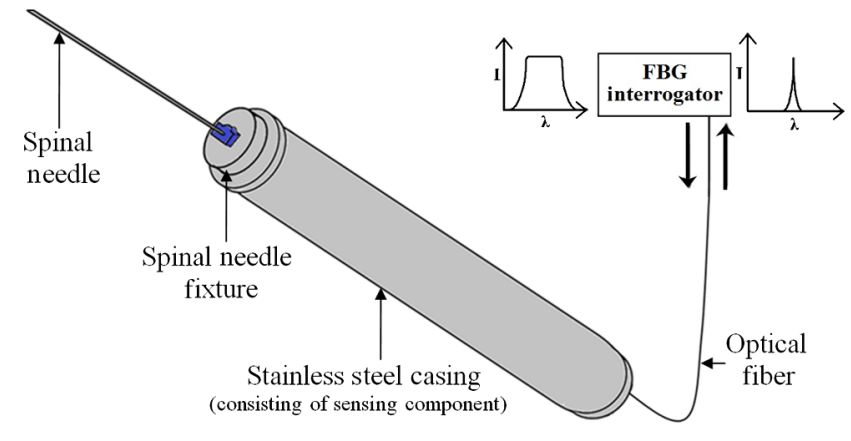

Fig. 2 Schematic of FBGFD with placement of spinal needle.

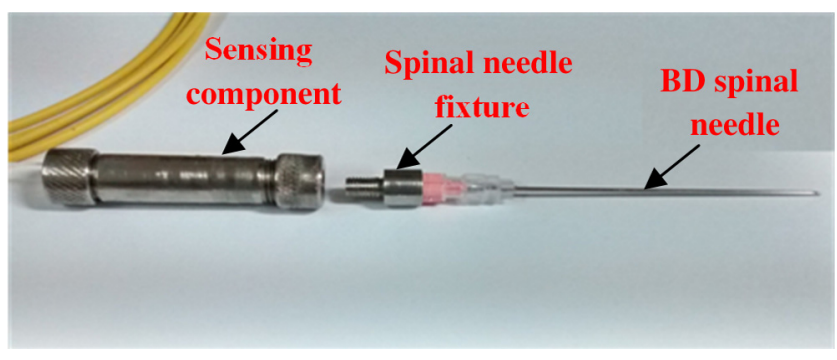

Fig. 3 Pictorial view of components of FBGFD.

Upon application of any force on the spinal needle mounted over the spinal needle fixture which is attached to the sensing component, the compressive force will be axially translated via the spinal needle fixture to the sensing component which further induces a compressive strain on the sensing bar. The FBG sensor acquires the compressive strain in terms of shift in Bragg wavelength. The lateral movement of the sensing component due to the spinal needle bending is negated by virtue of hollow tube walls. The reflected Bragg wavelength is acquired by means of micron optics interrogator SM130-700 which has a resolution of 1-pm wavelength shift. The complete system of the spinal needle fixture attached to the sensing component bonded with the FBG sensor housed inside a hollow casing constitutes to become the FBGFD. This is used with a mounted spinal needle for real-time dynamic monitoring of force acting on a spinal needle as shown in Fig. 2.

Thus utilizing FBGFD force experienced by the spinal needle during LP procedure, different tissue regions of varying stiffness penetrated can be dynamically monitored negating the effect of spinal needle bending. All components of FBGFD are made of stainless steel material as shown in Fig. 3. The FBGFD is made lightweight, compact, and rugged facilitating its easy usage manually by the medical practitioner.

\section{Experimental Analysis}

\subsection{Calibration Test}

The FBGFD developed has been calibrated using a Mecmesin's micro-universal testing machine ( $\mu \mathrm{UTM})$ for a range of applied force. The FBGFD is allowed to stand upright on a fixture for compressive force measurement (Fig. 4). The spinal needle fixture is put in direct contact with the UTM force device. The UTM is programmed to travel vertically at a rate of $1 \mathrm{~mm} / \mathrm{min}$ with application of force on FBGFD from the spinal needle fixture end. The Bragg wavelength shift of the FBG

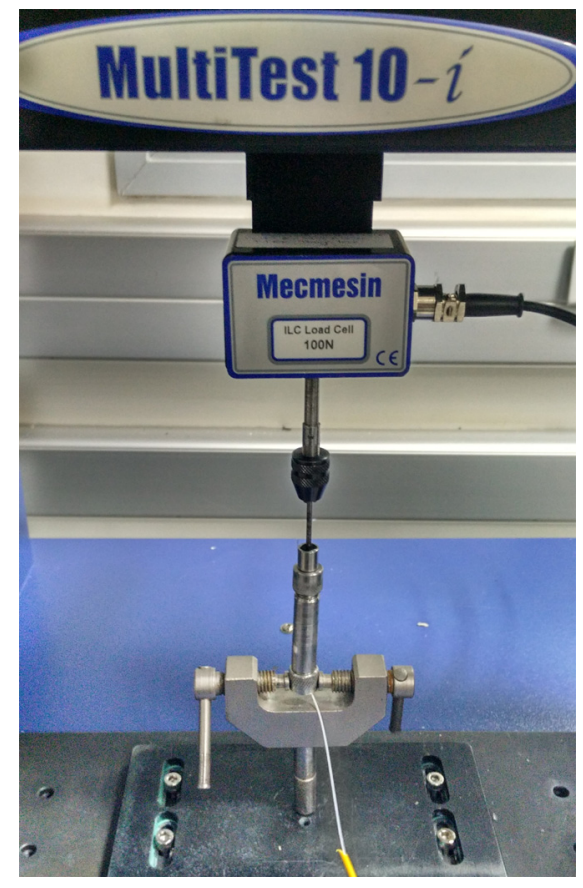

Fig. 4 FBGFD calibration setup.

sensor of FBGFD due to progressive application of force from UTM is continuously monitored and recorded.

Figure 5(a) shows the comparison of force monitored by UTM and the corresponding strain from the FBG sensor. It can be deduced that both FBG response and UTM response are in good agreement with each other in the investigated ranges of force. Further, for the range of forces applied by UTM, the strain experienced by FBGFD is obtained from the shift in the Bragg wavelength of the FBG sensor, using the strain sensitivity of $1.2 \mathrm{pm} / \mu \varepsilon{ }^{35}$ The shift in wavelength and the strain experienced by the FBGFD, with the application of UTM force, exhibits a linear response which is demonstrated by the correlation coefficient of 0.99 obtained as shown in Fig. 5(b). For a maximum applied force of $19.3 \mathrm{~N}$, the compressive strain in the FBGFD is found to be $733 \mu \varepsilon$, indicating an FBGFD sensitivity of $-38 \mu \varepsilon / \mathrm{N}$. The force sensitivity of FBGFD in terms of wavelength shift is obtained as $-46.4 \mathrm{pm} / \mathrm{N}$. The FBGFD sensitivity is dependent upon the thickness of the sensing bar. The sensitivity of the device reduces as the thickness of the sensing bar increases. The device has a fine resolution of $0.021-\mathrm{N}$ force measurement.

\subsection{Experimental Methodology}

An attempt is made to quantify the forces experienced by a spinal needle while it is introduced into the subarachnoid space of the Thoraco-lumbar spine. The experiment is carried out on a specially embalmed human cadaver specimen that has retained the organoleptic properties and gives the feel of live tissue while handling. The Cadaver specimen has been positioned in the right lateral position and the interspinous spaces have been palpated at the Thoraco-lumbar junction and the lumbar spine. The spinal needle with Quincke type point from Becton, Dickinson and Company (BD) of 18GA 3.50IN $(1.2 \mathrm{~mm} \times 90 \mathrm{~mm})$ is mounted to the FBGFD and introduced into the subarachnoid space as shown in Fig. 6(a). The dynamic force variations experienced by the spinal needle due to the resistance offered by various 


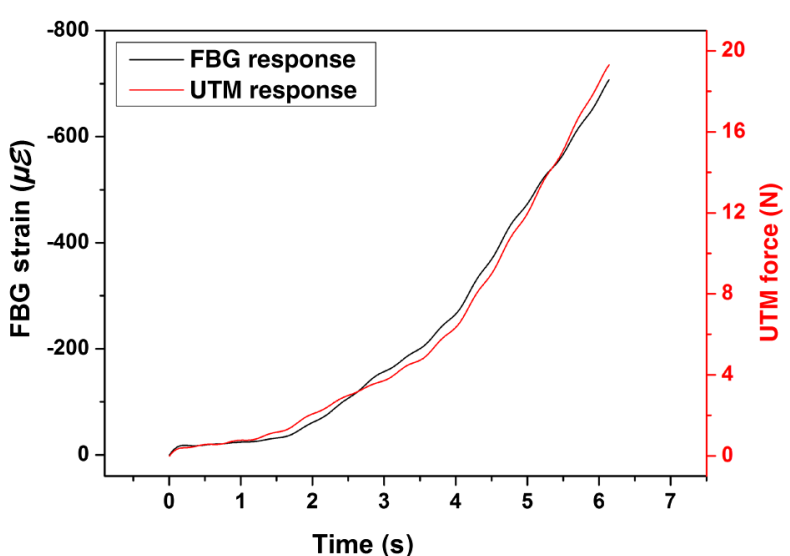

(a)

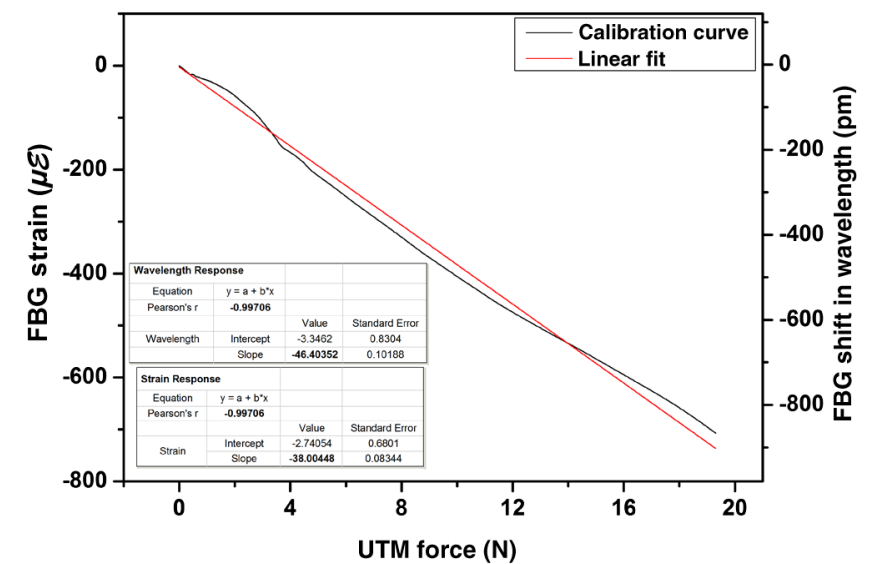

(b)

Fig. 5 (a) FBG and UTM response of FBGFD calibration and (b) FBGFD calibration curve.

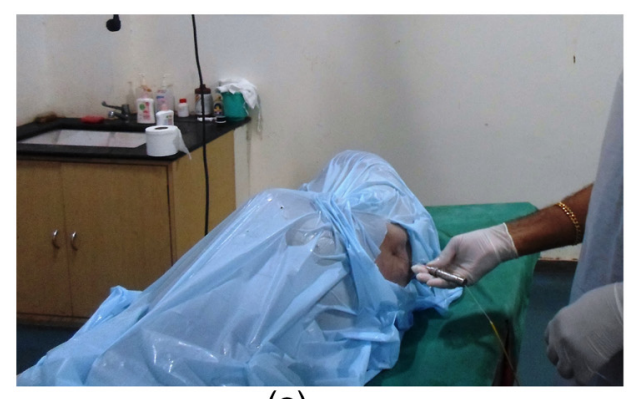

(a)

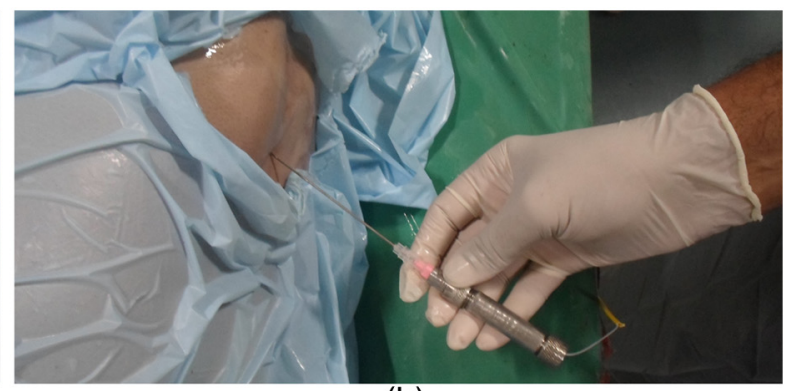

(b)

Fig. 6 Cadaver LP (a) experimental procedure with FBGFD and (b) closer view.

tissue layers are recorded from FBGFD during the spinal needle traversal. Multiple attempts of puncturing with the spinal needle are made with 18 gauge spinal needles at multiple levels from T12-L1 to L4-L5.

As the spinal needle penetrates through different tissue layers, the force experienced by it varies with the resistance due to tissue stiffness or in other words the force required to penetrate through a tissue layer depends on its stiffness. When the spinal needle traverses from regions of tissues with lower stiffness to higher stiffness, the tissue resistance increases which generates higher compressive force on the spinal needle recorded by the FBGFD and vice versa. The repeated tests and trials have been conducted with spinal needle insertion in different directions and pace in order to monitor the varying forces on the spinal needle.

\section{Results}

The validation experiments are carried out by an experienced medical practitioner by progressive insertion of the spinal needle, while performing an LP procedure on a human cadaver specimen; during the procedure, the dynamic force variations experienced by the spinal needle is recorded from the FBGFD. The dynamic force variations over the spinal needle are converted to strain variation and continuously recorded from the FBGFD during the LP procedure.

The varying force experienced by the spinal needle upon full length traversal through skin to epidural space puncturing various tissue layers during LP is shown in Fig. 7. The force response from the FBGFD is quantified with respect to time during the performance of the LP. Before the insertion of the spinal needle into the lumbar area, there is no force experienced on the needle as observed by the $0-\mathrm{N}$ force response. Compressive force monitored during spinal needle traversal inside the specimen is marked with the dotted lines. The spinal needle insertion initiates with the encounter of skin opposing its penetration with a resistive force creating a compressive force on it. A peak force of $4.27 \mathrm{~N}$ is recorded at which skin punctures allowing the needle to enter the cadaver specimen, as observed in Fig. 7. With continued traversal of the spinal needle, the force experienced

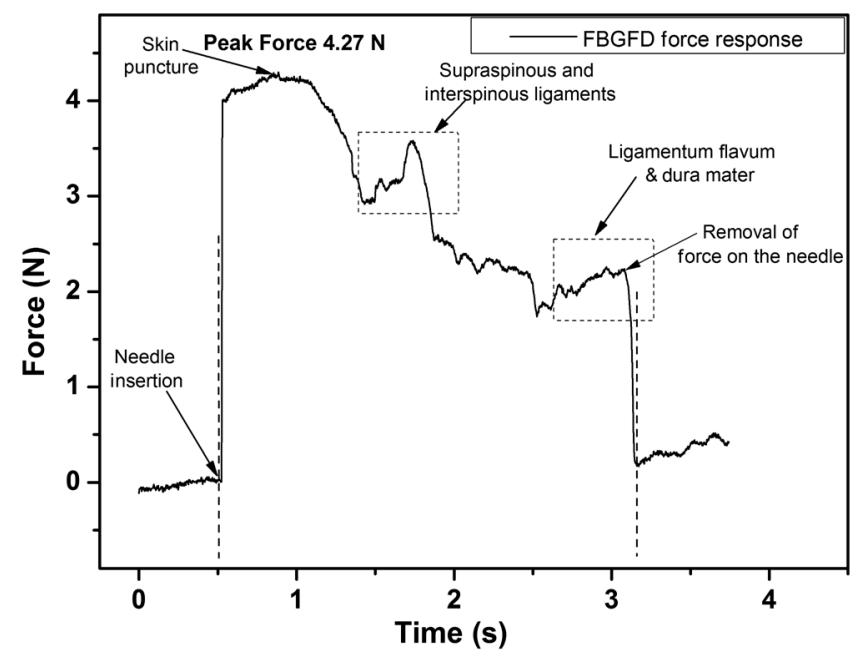

Fig. 7 Force experienced by spinal needle during tissue penetration recorded using FBGFD. 




Fig. 8 Force experienced by spinal needle during bone encounter recorded using FBGFD.

on it decreases due to the lesser resistance offered by the tissue layers beneath the skin layer.

Further, the spinal needle at the supaspinous and interspinous ligament regions experiences higher resistance and hence a force of $3.5 \mathrm{~N}$ is recorded during piercing this region. The sudden decrease in the force on the spinal needle as observed in Fig. 7 indicates the specific tissue being punctured and the spinal needle's progression into the next tissue layer. As the spinal needle traverses further the resistance offered by the tissue layers are lower when compared to the initial tissue layers beneath the skin. This shows that the tissue layers beneath the skin are stiffer than the tissue layers in the supaspinous and interspinous ligament regions. A force of $2.2 \mathrm{~N}$ is recorded on the spinal needle which represents the piercing of the ligamentum flavum and dura mater and the entrance of the spinal needle into the epidural space, as observed from Fig. 7. Upon reaching the epidural space, the resistance offered to the spinal needle drastically decreases, which is also sensed by the medical practitioner who is performing the procedure. As the spinal needle enters the epidural space, the insertion process is stopped which is observed as the force on the spinal needle returning toward the zero baseline, depicting the end of the LP procedure.

Direction of spinal needle insertion and its traversal is a key factor during the LP test in order to find an optimized pathway of its traversal avoiding encounter of unwanted bones, organs, or tissues, minimizing accidental injuries. Another LP test performed on the same cadaver specimen, from different directions is depicted in Fig. 8. It is observed that the force on the spinal needle increases with the traversal after the puncturing of the skin layer. The force on the spinal needle increases to 11.45 $\mathrm{N}$, which is due to the bone encountered in the traversal path of the spinal needle. The bone does not allow the spinal needle to pierce through and hence a high opposing force is observed as indicated by trial 1 in Fig. 8. As no further insertion of the spinal needle is possible, the medical practitioner had to retrace the needle and begin the procedure again in a different direction.

During the reinsertion of the spinal needle shown as trial 2 in Fig. 8, the application of force for skin puncturing is carried out at a slower rate. The spinal needle punctures the skin with a force of $4.31 \mathrm{~N}$ which is in good agreement with the earlier test performed. Also, the penetration of regions of supaspinous and interspinous ligament along with ligamentum flavum and dura mater by the spinal needle is observed with a force of 2.2 and $0.8 \mathrm{~N}$, respectively. The resistance offered by the tissue regions to the traversal of the spinal needle also depends on the direction of the spinal needle insertion. Therefore, the forces required to penetrate these regions in the opted pathway of trial 2 are observed to be less than the earlier tests performed. Upon reaching the epidural space, the insertion procedure is halted and the force on the spinal needle returns to the baseline state. The undesirable bone encounter can be avoided upon comprehending a high spinal needle force on real time using FBGFD.

\section{Discussion}

These obtained results proved the efficacy of the developed FBGFD's employment as a force feedback mechanism during the LP tests. The FBGFD responds only to compressive force acting on the spinal needle negating any bending effect along with any vibrations generated during the insertion procedure. Also, chances of the sensor getting damaged are greatly reduced because of the rugged and enclosed structure of FBGFD. The FBG sensor is present inside the FBGFD and is not in direct contact with the specimen body or the surgeon performing the LP procedure. Thus, the FBG sensor is not affected by any temperature change; hence no temperature compensation is required. This demonstrates its usability in clinical applications for realtime force monitoring during spinal needle insertion procedures.

It is important to note that the FBGFD is devised providing the possibility for sterilization of the detachable spinal needle fixture. The FBGFD can be reused for LP tests with the replacement of the spinal needle. The continuous feedback force monitoring of the spinal needle traversal helps in correctly determining the regions penetrated and also preventing any unwanted further penetration of the spinal needle upon reaching the region looked-for. As the spinal needle reaches the desired location in the epidural space, the FBGFD can be detached releasing the spinal needle. Thus, only the spinal needle is left, from which a sample of CSF may be extracted from the epidural space for testing and for any further procedures required in surgery. Furthermore, FBGFD with its feedback force monitoring capability may greatly aid in providing a safe method for trainees to perform the LP procedure, knowing the region of spinal needle penetration.

\section{Conclusion}

A FBGFD, based on an FBG sensor, for the real-time dynamic spinal needle force monitoring, having a resolution of $0.021 \mathrm{~N}$, has been presented in this work. Design and development of the FBGFD and its validation in an LP test are detailed. The FBGFD has an ability to mount spinal needles of various gauge size for carrying out LP procedures.

The resistances experienced due to various tissue regions of varying stiffness encountered while inserting the spinal needle is axially translated into strain which is acquired by the FBG sensor. The resultant compressive force acting on the spinal needle is evaluated which in turn is the coarse indication of the region of tissue layer being pierced by the spinal needle. Various layers encountered by an 18-GA spinal needle during experiments performed on a human cadaver specimen have been illustrated in the form of varying forces for the spinal needle traversal.

The present device can be potentially used to provide realtime tactile feedback for a successful LP avoiding any severe surgical accident. The FBGFD has an assembly of parts that can be repeatedly utilized. Further, the present device can be used to examine the forces experienced by different gauges of 
spinal needles using FBGFD, during LP tests. The authors declare that there is no conflict of interest.

\section{References}

1. B. Tang, G. B. Hanna, and A. Cuschieri, "Analysis of errors enacted by surgical trainees during skills training courses," Surgery 138, 14-20 (2005).

2. P. Gorman et al., "A prototype haptic lumbar puncture simulator," in Medicine Meets Virtual Reality, J. D. Westwood et al., Eds., pp. 106-109, IOS Press, Amsterdam (2000).

3. A. P. Daykin and R. J. Bacon, "An epidural injection simulator," Anaesthesia 45, 235-236 (1990).

4. A. M. Okamura, C. Simone, and M. D. O'Leary, "Force modeling for needle insertion into soft tissue," IEEE Trans. Biomed. Eng. 51(10), 1707-1716 (2004).

5. J. Z. Moore et al., "Hollow needle tissue insertion force model," CIRP Ann. Manuf. Technol. 60, 157-160 (2011).

6. L. L. H. Holton, "Force models for needle insertion created from measured needle puncture data," in Medicine Meets Virtual Reality, J. D. Westwood et al., Eds., pp. 180-186, IOP Press, Amsterdam (2001).

7. H. Kataoka et al., "Measurement of the tip and friction force acting on a needle during penetration," in MICCAI 2488, T. Dohi and R. Kikinis, Eds., pp. 216-223, Springer-Verlag, London (2002).

8. N. Vaughan et al., "A review of epidural simulators: where are we today?" Med. Eng. Phys. 35, 1235-1250 (2013).

9. K. W. Ng et al., "Needle insertion forces studies for optimal surgical modeling," Int. J. Biosci., Biochem. Bioinf. 3, 187-191 (2013).

10. B. Gillespie and L. B. Rosenberg, "Design of high-fidelity haptic display for one-dimensional force reflection applications," Proc. SPIE 2351, 44-54 (1994).

11. N. Abolhassani, R. Patel, and M. Moallem, "Needle insertion into soft tissue: a survey," Med. Eng. Phys. 29, 413-431 (2007).

12. D. S. Kwon et al., "Realistic force reflection in a spine biopsy simulator," in Proc. IEEE Int. Conf. on Robotics \& Automation, Seoul, Korea, 1358-1363 (2001).

13. M. Farber, J. Heller, and H. Handels, "Simulation and training of lumbar punctures using haptic volume rendering and a 6DOF haptic device," Proc. SPIE 6509, 65090F (2007).

14. G. Tholey, J. P. Desai, and A. E. Castellanos, "Force feedback plays a significant role in minimally invasive surgery-results and analysis," Ann. Surg. 241, 102-109 (2005).

15. A. R. Lanfranco et al., "Robotic surgery: a current perspective," Ann. Surg. 239, 14-21 (2004).

16. J. C. Magill et al., "A novel actuator for simulation of epidural anesthesia and other needle insertion procedures," J. Soc. Simul. Healthcare 5(3), 179-184 (2010).

17. P. N. Brett et al., "Automatic surgical tools for penetrating flexible tissues," IEEE Eng. Med. Biol. Mag. 14(3), 264-270 (1995).

18. M. Bostrom, S. K. Singh, and C. W. Wiley, "Design of an interactive lumbar puncture simulator with tactile feedback," in IEEE Virtual Reality Annual International Symp., Seattle, Washington, pp. 280-286 (1993).

19. T. Dang, T. M. Annaswamy, and M. A. Srinivasan, "Development and evaluation of an epidural injection simulator with force feedback for medical training," in Medicine Meets Virtual Reality, J. D. Westwood et al., Eds., Vol. 81, pp. 97-102, IOP Press, Amsterdam (2001).

20. S. P. DiMaio and S. E. Salcudean, "Needle insertion modeling and simulation," IEEE Trans. Robot. Automat. 19, 864-875 (2003).

21. S. Umesh and S. Asokan, "A brief overview of the recent bio-medical applications of fiber Bragg grating sensors," J. Indian Inst. Sci. 94(3), 319-328 (2014).

22. A. Mendez, "Fiber Bragg grating sensors for biomedical applications," in OSA Photonics and Fiber Technology Congress (2016).

23. E. Al-Fakih, N. A. A. Osman, and F. R. M. Adikan, "The use fiber Bragg grating sensors in biomechanics and rehabilitation applications: the state-of-the-art and ongoing research topics," Sensors 12, 1289012926 (2012).

24. P. Roriz et al., "Review of fiber-optic pressure sensors for biomedical and biomechanical applications," J. Biomed. Opt. 18(5), 050903 (2013).
25. Q. Yu et al., "Study on optical fiber Bragg grating temperature sensors for human body temperature monitoring," in Proc. Symp. Photonics and Optoelectronics (SOPO), pp. 1-4 (2012).

26. J. Paul, L. Zhao, and B. K. A. Ngoi, "Fiber optic sensor for handgripstrength monitoring: conception and design," Appl. Opt. 44(18), 36963704 (2005).

27. D. H. C. Wang et al., "An optical fiber Bragg grating force sensor for monitoring sub-bandage pressure during compression therapy," Opt. Express 21(17), 19799-19807 (2013)

28. A. Othonos, "Fiber Bragg gratings," Rev. Sci. Instrum. 68, 4309-4341 (1997).

29. K. O. Hill et al., "Bragg gratings fabricated in monomode photosensitive optical fibers by UV exposure through a phase mask," Appl. Phys. Lett. 62, 1035-1037 (1993).

30. A. Othonos and K. Kalli, Fiber Bragg Gratings Fundamentals and Applications in Telecommunications and Sensing, Artech House, Boston, (1999).

31. R. Kashyap, Fiber Bragg Gratings, Academic Press, San Diego, (1999).

32. K. O. Hill and G. Meltz, "Fiber Bragg grating technology fundamentals and overview," J. Lightwave Technol. 15, 1263-1276 (1997).

33. G. Meltz, W.W. Morey, and W. H. Glam, "Formation of Bragg grating in optical fibers by a transverse holographic method," Opt. Lett. 14, 823-825 (1989).

34. B. A. Tahir, J. Ali, and R. A. Rahman, "Fabrication of fiber grating by phase mask and its sensing application," J. Optoelec. Adv. Mat. 8(4), 1604-1609 (2006).

35. S. M. Melle et al., "A Bragg grating-tuned fiber laser strain sensor system," IEEE Photonics Technol. Lett. 5(2), 263-266 (1993).

Shikha Ambastha received her MTech degree in measurement and instrumentation from Indian Institute of Technology, Roorkee, India, in 2010. She joined CSIR-CMERI, Durgapur, India, as a scientist in 2010 and worked in the field of robotics and automation. Currently, she is pursuing a doctoral degree at the Fiber Bragg Grating Sensors Laboratory, Department of Instrumentation and Applied Physics, Indian Institute of Science. Her current research interest includes FBG sensors applications for biomedical sensing and engineering application, and sensor packages for robotic applications.

Sharath Umesh received his doctorate from the Department of Instrumentation and Applied Physics, Indian Institute of Science, Bangalore, India, in 2016. He received his BE degree in mechanical engineering from Visvesvaraya Technological University, India, in 2009. He is the recipient of the Prof. K. N. Amulya Reddy Award among the Gandhian Young Technological Innovation Awards 2015. $\mathrm{He}$ is currently working as a research associate at the Indian Institute of Science.

Sundaresh Dabir received his MBBS degree from Manipal University, India, and his master's degree in orthopedics from KMC Manipal, India. He has been the HOD at the Ramaiah Hospital and an orthopedic surgeon as well as president of the Advanced Learning Centre and pro vice-chancellor of the M S Ramaiah University of Applied Sciences. He has served as the chairman and senior consultant, Orthopedic Department at Sri Sathya Sai Institute of Higher Medical Sciences, Prasanthigram. Currently, he is the director of the Sri Sathya Sai Institute of Higher Medical Sciences, Whitefield, Bangalore, India.

Sundarrajan Asokan is currently a professor and chairman of the Department of Instrumentation and Applied Physics, Indian Institute of Science, India. He is the recipient of the Martin J. Forster Gold Medal of the Indian Institute of Science for the best $\mathrm{PhD}$ thesis in the Division of Physical and Mathematical Sciences, the Young Scientist Medal of the Indian National Science Academy in 1990, and the Young Scientist Research Award of the Department of Atomic Energy in 1995, India. He has been elected a fellow of the National Academy of Sciences, India, in 2008. 